\title{
A recessively inherited ataxia with episodes of dystonia
}

\author{
NEILL R GRAFF-RADFORD \\ From the Department of Neurology, University of Iowa College of Medicine, Iowa City, Iowa, USA
}

SUMMARY A family with recessively inherited ataxia and dystonic episodes that responded to antiepileptic medication is described. The onset was in the first decade. Clinically the patients have gait and limb ataxia, nystagmus and brisk reflexes, with abnormal visual, auditory and somatosensory evoked responses, but normal nerve conduction velocities and electromyography. Their intelligence is borderline. CT and MRI scans show severe atrophy in the vermis and basis pontis.

Recessively inherited progressive ataxias of childhood onset may be caused by a known metabolic abnormality such as abetalipoproteinemia, ${ }^{1}$ hypobetalipoproteinemia, ${ }^{1}$ hexosaminidase deficiency, ${ }^{2}$ and cholestanolosis. ${ }^{3}$ However, there is still a large group without established metabolic abnormalities. ${ }^{4}$ In one study of ataxias there was a group with progressive unremitting ataxias of childhood onset, characterised by having brisk reflexes. ${ }^{5}$ In this paper, a family believed to be a subgroup of the group described in the above study is reported. In this family both parents are normal but all three siblings are affected by an unremitting progressive ataxia of childhood onset. All the siblings have brisk reflexes. However, this family's symptom complex is distinguishable also by including intermittent disabling dystonic episodes.

\section{Case reports}

Case I

A 21-year-old right-handed, non-Jewish woman, was referred to the University of Iowa Hospitals for progressive difficulty in walking, clumsiness and attacks of muscle tightening. She was the product of a normal pregnancy and uncomplicated birth and her parents are unrelated. Her developmental milestones were normal until age 4 years. Between the ages of 4 and 6 her parents noted that her running was awkward and hand coordination clumsy. There

Address for reprint requests: Dr Neill R Graff-Radford, Department of Neurology, University of Iowa Hospitals \& Clinics, Iowa City, Iowa 52242, USA.

Received 18 June 1985 and in revised form 4 September 1985 Accepted 10 September 1985 has been a slowly progressive ataxia until the present. She can still walk independently, but prefers to use a wheelchair. Over the last six years she has also complained of attacks of muscle tightening. She describes these as a sudden tightening of her body, more on the left than the right. The attacks last from 30 seconds to several minutes. Although she is fully conscious during the attacks, she cannot speak because of the tightening of her facial, pharyngeal and laryngeal muscles. There have been no associated movements or incontinence. Off medication she may have more than 20 attacks a day. Phenytoin diminished the attacks but made her much more ataxic. Carbamazepine also decreased the attack frequency. Meclizine, acetazolamide and diphenhydramine had no affect. Her general examination was normal. There was no evidence of thickened tendons, cataracts, pes cavus or scoliosis. Her fundi, acuity and visual fields were normal. She had nystagmus on lateral gaze. The rest of her cranial nerves were normal but she was dysarthric. Her reflexes were brisk, with bilateral ankle clonus and an extensor plantar response on the right. Her strength and sensory examination were normal. She performed poorly on the finger/nose, rapid alternating hand movements and heel/knee shin tests. Her gait was wide-based and ataxic. I witnessed several of her attacks. During one her face pulls towards the left side while her left arm becomes straight and stiff by her side and her left leg stiffens with inversion at the ankle. The changes build up over a few seconds and dissipate in about one minute. The patient remains immobile and aphonic, standing and holding on for support. There is no post-ictal confusion. The patient had a normal blood count, serum electrolytes, liver function tests, caeruloplasmin, protein electrophoresis and hexosaminidase. She underwent skin biopsy from which fibroblasts were cultured. These were assayed for glutamate dehydrogenase which was normal. An electroencephalogram showed no seizure activity during several spells. Nerve conduction and electromyography were normal. Brain stem auditory evoked responses were abnormal with absence of all 
waves after wave I. Visual evoked responses were abnormal with P100 delayed to $163 \mathrm{~ms}$ in the left eye and $155 \mathrm{~ms}$ in the right eye. Sensory evoked responses were delayed after the brain stem P $\overline{14}$. Neuropsychological evaluation showed that the patient performed in the borderline range on tests of intellectual ability; WAIS-R verbal IQ was 85 and performance IQ 76. Verbal memory and visual memory for designs was normal. She was not aphasic. Her academic achievement tests revealed abilities in oral word reading (39th percentile), spelling (50th percentile), and arithmetic (32nd percentile). Her CT and MRI scans revealed significant cerebellar atrophy, particularly of the vermis, as well as atrophy of the pons (fig a).

\section{Case 2}

The first patient's 22-year-old right-handed brother was also evaluated at the University of Iowa Hospitals. He was the product of a normal pregnancy and uncomplicated birth. His motor milestones were normal but at about age 6 years his parents noted that he was clumsy and had difficulty with sport activities and running. He also slurred his speech. The patient had attacks similar to, but less frequent than, his sister. Phenytoin and carbamazepine decreased the number of attacks. His general examination was normal. He had no tendon thickening, cataracts, pes cavus or scoliosis. His fundi, visual fields and acuity were normal. There was nystagmus on lateral gaze. His reflexes were brisk with sustained clonus at the ankles. Plantar responses were flexor. Strength and sensory examination were normal. He performed poorly on the finger/nose, rapid alternating movements and heel/knee/shin tests. His gait was wide-based and ataxic. He had normal blood count, serum electrolytes, liver function tests, protein electrophoresis and hexosaminidase. Cultured skin fibroblasts were tested for glutamate dehydrogenase which was normal. Nerve conduction velocities and electromyography were normal. Brain stem auditory evoked responses. showed an absence of peaks after wave 1. Visual evoked responses showed that $\mathrm{P} \overline{100}$ was delayed in both eyes, the left was $127 \mathrm{~ms}$ and the right $125 \mathrm{~ms}$. Sensory evoked responses showed a delay in N19. The delay possibly occurred between $\mathrm{N} \overline{13}$ and $\mathrm{P} \overline{14}$ and between $\mathrm{N} \overline{17}$ and $\mathrm{N} \overline{19}$. On neuropsychological evaluation his intellectual ability was in the low average range. WAIS-R verbal IQ $=91(27$ th percentile) and performance IQ $=80$ (9th percentile). Verbal memory and visual memory for designs were in the normal range. The patient was not aphasic. Assessment of academic achievement levels revealed average oral word reading (30th percentile) but impaired spelling (4th percentile) and arithmetic (5th percentile). CT and magnetic resonance imaging showed significant atrophy of the cerebellum, especially of the vermis and of the pons (fig b).

\section{Case 3}

We also evaluated the third sibling of the family at the University of Iowa Hospitals. He was a 17-year-old righthanded man, again the product of a normal pregnancy and birth. His milestones were also normal, but he was noted to be clumsier than other children when he started school. He had a normal general examination. On neurological examination, he too had dysarthria, nystagmus, brisk reflexes, incoordination and gait ataxia.

Both parents were evaluated and found to be normal.

\section{Discussion}

This family falls into the group of progressive, childhood onset recessively inherited ataxia with brisk reflexes. The cases differ from those previously reported by having episodic dystonia of almost the entire body, albeit asymmetric. These responded to antiepileptic drugs. Two previous cases of dystonia associated with ataxia have been reported. In the first van Bogaert et $a l^{6}$ described a 3 -year-old child with ataxia and continuous dystonic posture associated with anosmia. In the second, Mayeux and Fahn ${ }^{7}$ described a patient with episodes of dystonia in the setting of progressive ataxia. Their patient differs from ours by having a normal CT and probably being of dominant inheritance in that the grandfather, uncle, mother and brother were affected. In addition, phenytoin increased the number of attacks while clonazepam decreased the frequency. Phenytoin decreased the frequency of our patients' dystonic attacks.

Our patients do have some similarity to patients with familial paroxysmal dystonic choreoathetosis, ${ }^{8}$ also called familial and acquired paroxysmal dyskinesia. ${ }^{9}$ This is a group of disorders which may be familial or acquired. The acquired causes, as referenced by Lance, $^{8}$ are cerebral palsy, a cortical scar, multiple sclerosis and basal ganglia disease. If familial the attacks may be precipitated by movement, in which case they are called kinesigenic and if not, nonkinesigenic. Patients with the kinesigenic form usually respond to anticonvulsants but anticonvulsants may make the non-kinesigenic form worse. In most patients with the familial form an autosomal dominant pattern of transmission is reported and the neurological examination is usually normal. While it is important to contrast our patients with this group of diseases, because they have similar attacks to some patients with the non-kinesigenic form, our patients differ by responding to anticonvulsants, probably having a recessive form of inheritance and having a degenerative brain disease with a severe ataxia.

The CT and MRI scans in our patients indicate that the brunt of the degenerative process affects the vermis of the cerebellum and the basis pontis. However, other areas of the brain are also probably affected as evidenced by the delay in visual evoked responses, borderline intelligence and perhaps slightly enlarged ventricles for age. Pathological examination would be needed to confirm all the areas affected. Normal nerve conduction velocities and electromyography make a peripheral nervous system component unlikely.

While the family probably does have a metabolic abnormality, which is found in recessively inherited childhood onset ataxias, we have been unable to establish the specific abnormality. The clinical features are unlike abetalipoproteinemia, ${ }^{1}$ hypo- 


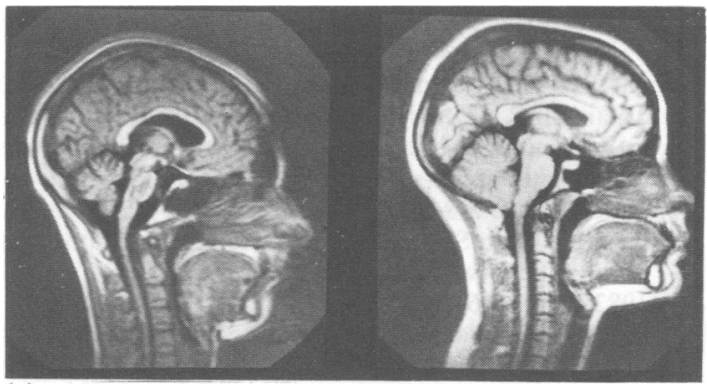

Fig (a) Midsagittal view of magnetic resonance scan in patient 1 (left) and age and sex matched normal volunteer (right). Note vermis atrophy, atrophy of the pons with enlargement of the prepontine cistern and an enlarged fourth ventricle. (b) Four $\mathrm{mm} \mathrm{CT}$ slices in patient 2. Note vermis atrophy with an enlarged fourth ventricle and cisterna magna. There is also pontine atrophy with an associated enlargement of the prepontine cistern. The ventricles are enlarged for his age.

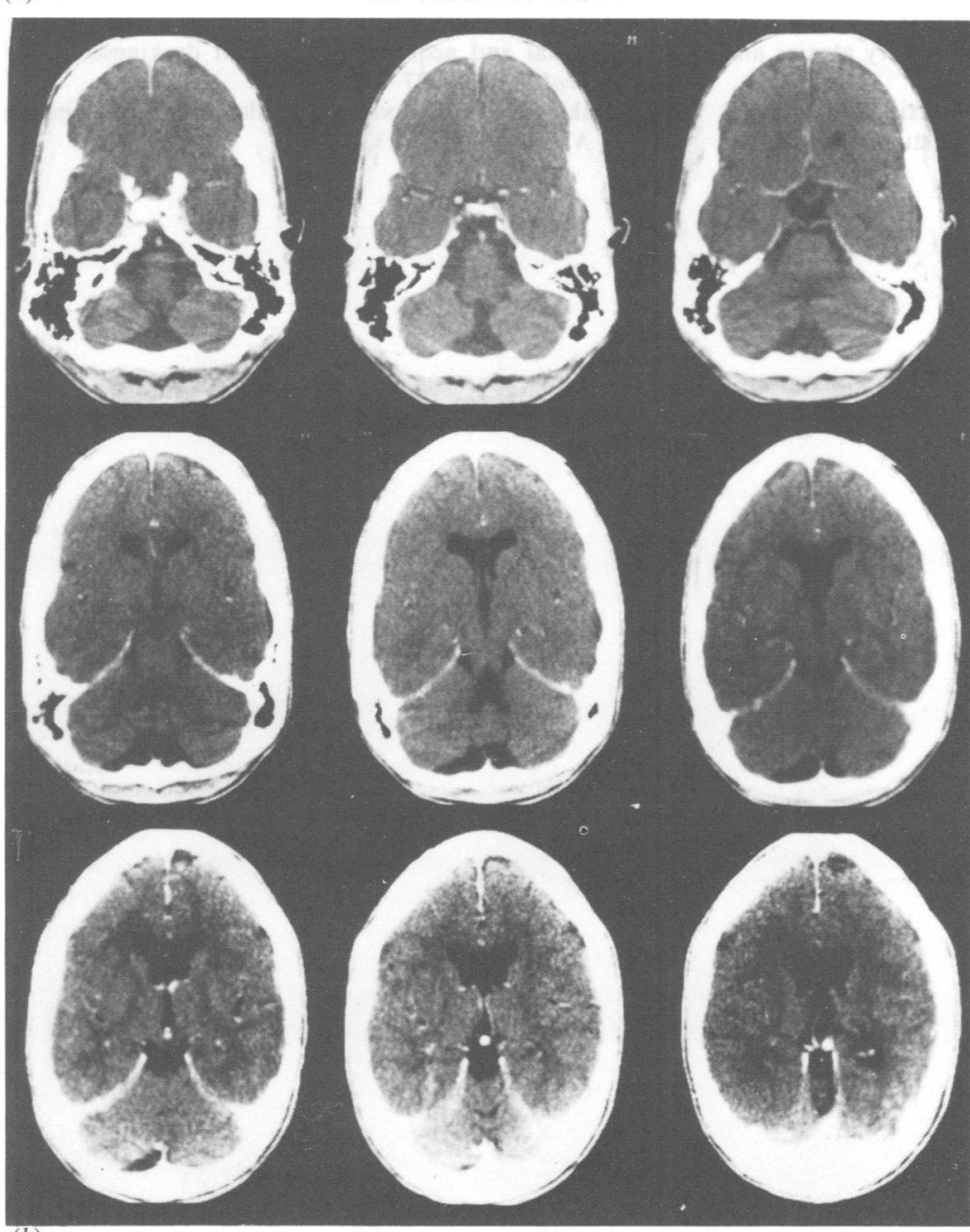

(b)

betalipoproteinemia, ${ }^{1}$ hexosaminidase deficiency, ${ }^{2}$ cholestanolosis, ${ }^{3}$ and glutamate dehydrogenase deficiency. ${ }^{10}$ In addition, we assayed glutamate dehydrogenase on cultured fibroblasts which was normal. The normal size tendons and absence of cataracts are against cholestanolosis.
I thank Ms Jean Hulme for help with the preparation of this manuscript, Dr Thoru Yamada for interpreting the EEG and evoked responses, Dr Val Dunn for interpreting the MRI scan, Dr AJ Plaitakis for assaying the glutamate hydrogenase and Dr GA Grabrowski for assaying the hexosaminidase. 


\section{References}

${ }^{1}$ Herbert PN, Gotto AM, Frederickson DS. Familial lipoprotein deficiency. In: Stanbury JB, Wyngaarden JB, Frederickson DS, eds. New York: McGraw Hill 1978:544-88.

${ }^{2}$ Willner JP, Grabowski GA, Gordon RE, Bender AN, Desnick RJ. Chronic $\mathrm{GM}_{2}$ gangliosidosis masquerading as atypical Friedreich ataxia: clinical, morphologic and biochemical studies of nine cases. Neurology (NY) 1981;31:787-98.

${ }^{3}$ Farpour H, Mahloudji M. Familial cerebrotendinous xanthomatosis. Arch Neurol 1975;32:223-5.

${ }^{4}$ Harding AE. Classification of the hereditary ataxias and paraplegias. Lancet 1983;i:1151-5.

${ }^{5}$ Harding AE. Early onset cerebellar ataxia with retained tendon reflexes: a clinical and genetic study of a disorder distinct from Friedreich's ataxia. $J$ Neurol Neurosurg Psychiatry 1981;44:503-8.

${ }^{6}$ Bogaert L van, David M, de Ajuriaguerra J, Hecaen $H$, Talairach R. D'un syndrome degeneratif du groupe de la dystonie a debut cerebelleux correspondent a une abiotrophie spino-striee avec degenerescence du systeme olfactif. Mschr Psychiat Neurol 1951;121:143-62.

${ }^{7}$ Mayeux F, Fahn S. Paroxysmal dystonic choreoathetosis in a patient with familial ataxia. Neurology $(N Y)$ 1982;32:1184-6.

${ }^{8}$ Lance JW. Familial paroxysmal dystonic choreoathetosis and its differentiation from related syndromes. Ann Neurol 1977;2:285-93.

${ }^{9}$ Goodenough DJ, Fariello RG, Annis BL, Chun RWM. Familial and acquired paroxysmal dyskinesias. Arch Neurol 1978;35:827-31.

${ }^{10}$ Plaitakis A. The olivopontocerebellar atrophies. Seminars in Neurology 1982;2:334-42. 